\title{
SÍNTESE CONTÍNUA DE BIODIESEL POR TRANSESTERIFICAÇÃO NÃO CATALÍTICA DE ÓLEO DE PINHÃO MANSO EM ETANOL
}

\author{
Bruna Cruzeiro Lopes ${ }^{1}$, Jéssica de Carvalho Lima'1 ${ }^{1}$ Alexandre Teixeira de Souza² \\ 1Discente e 2Docente do Curso de Engenharia Ambiental da UNOESTE, Presidente Prudente, SP.
}

\section{RESUMO}

Este projeto teve a proposta viabilizar a obtenção do biodiesel utilizando o óleo extraído da Jatropha curcas L., também conhecida como pinhão manso, através da transesterificação contínua e não-catalítica utilizando etanol em condições pressurizadas. As análises experimentais visaram avaliar a influência das variáveis de processo tais como: temperatura, razão molar de óleo para álcool e tempo de residência sobre a conversão da reação em termos de ésteres etílicos de ácidos graxos, objetivando o estabelecimento de condições amenas de operação e garantia de qualidade do produto gerado. O método supercrítico apresenta facilidade de separação dos produtos da reação, tornando a purificação do biodiesel mais fácil e sustentável, redução considerável do tempo de reação e não apresenta restrição em relação ao teor de ácidos graxos livres e água presentes nos substratos, justificando a utilização da presente metodologia, para a obtenção de biodiesel proveniente de óleo de pinhão manso.

Palavras-chave: biodiesel, transesterificação não catalítica, método supercrítico, óleo de pinhão manso.

\section{CONTINUED SYNTHESIS OF BIODIESEL BY TRANSESTERIFICATION NOT CATALYTIC OF JATROPHA OIL IN ETHANOL}

\section{ABSTRACT}

This project was proposed to facilitate the obtaining of biodiesel using oil extracted from Jatropha curcas L., through transesterification continuous catalytic and non pressurized conditions using ethanol. The experimental analysis aimed to evaluate the influence of process variables such as temperature, molar ratio of oil to alcohol and residence time on conversion of the reaction in terms of fatty acid ethyl esters, aiming at establishing the mild conditions of operation and warranty quality of the product generated. The supercritical method provides ease of separation of reaction products, making it easier purification of biodiesel and sustainable reduction in the time of reaction and has norestriction regarding the content of free fatty acids and water present in the substrates, justifying the use of this methodology to obtain biodiesel from jatropha oil.

Keywords: biodiesel, transesterification non-atalytic, supercritical method, jatropha oil. 


\section{INTRODUÇÃO}

A demanda mundial por combustíveis alternativos tem se expandido rapidamente, devido, principalmente, à necessidade de diminuir a dependência de combustíveis não-renováveis derivados de petróleo, e à crescente preocupação ambiental, visto que o uso do petróleo como fonte energética representa uma das maiores causas da poluição atmosférica, e sua combustão causa o aumento da concentração de dióxido de carbono na atmosfera, contribuindo assim para o indesejável efeito estufa.

Os combustíveis provenientes de fontes renováveis, como a biomassa, constituem uma das alternativas mais promissoras, principalmente nos países com grandes extensões territoriais e com clima propício para a atividade agrícola, como é o caso do Brasil.

O biodiesel (mistura de ésteres de ácidos graxos), derivado de triglicerídeos por transesterificação com um álcool, tem atraído considerável atenção como combustível renovável, biodegradável e não-tóxico, tendo grande potencial como alternativa ao Diesel de petróleo. Tais benefícios justificam o crescente interesse no desenvolvimento de tecnologias referentes à produção deste biocombustível em diversos países e no âmbito nacional, do ponto de vista tecnológico, econômico e ambiental.

A escolha da oleaginosa utilizada considerou o teor em óleo vegetal, produtividade, adaptação territorial (RAMOS et al., 2003). Observando-se os aspectos tecnológicos e econômicos, é de fundamental importância que se tenha uma visão geral da potencialidade dos óleos vegetais na produção de biodiesel, no âmbito nacional cada região apresenta potencialidade para diferentes tipos de oleaginosas.

A Jatropha curcas L., também conhecida como pinhão manso, pertence à família
Euphorbiaceae. É uma espécie nativa da América tropical e naturalizada em partes tropicais e subtropicais da Ásia e África (AUGUSTUS et al., 2002; ARRUDA et al., 2004). A distribuição geográfica do pinhão manso é bastante vasta devido à sua rusticidade, resistência a longas estiagens, bem como à pragas e doenças, sendo adaptável a condições edafoclimáticas muito variáveis. Desenvolve-se bem tanto em regiões tropicais secas como nas zonas equatoriais úmidas, assim como em terrenos áridos e pedregosos podendo suportar longos períodos de seca (ARRUDA et al., 2004; PRAMANIK et al., 2003).

Os frutos de J. curcas são utilizados no tratamento de doenças como disenteria, hemorróidas, gonorréia, infertilidade, infecções na pele, etc. (AKINTAYO, 2004). Apresentam ainda uma grande importância econômica, sendo o óleo empregado como lubrificante, na fabricação de sabão, tinta e combustível para motores diesel (OPENSHAW, 2000; SUBRAMANIAN et al., 2005; FOIDL et al., 1996).

Em função do potencial apresentado pelo pinhão manso, como fonte de matéria prima para produção de biodiesel e o Estado do Piauí possuir condições edafoclimáticas adequadas a seu cultivo, o presente trabalho, visou contribuir com pesquisas para viabilizar o processo de obtenção do biodiesel a partir dessa matriz oleaginosa, a fim de garantir um custo competitivo para sua produção.

Entre outros processos para produção de biodiesel, tais como: diluição, microemulsão, decomposição térmica e craqueamento catalítico, a transesterificação tem sido $\circ$ método mais utilizado atualmente (MA; HANNA, 1999; FUKUDA et al., 2001). Transesterificação, também chamada de alcoólise, refere-se à reação de um triglicerídeo (de fonte vegetal ou animal) com um álcool na presença ou ausência de 
catalisador, da qual resulta uma mistura de ésteres de ácidos graxos denominada biodiesel, e o glicerol como subproduto. Convencionalmente, a alcoólise de óleos vegetais pode ser conduzida por intermédio de catalisadores químicos (ácidos ou bases) ou enzimáticos (MA; HANNA, 1999; FUKUDA et al., 2001; DEMIRBAS, 2007; MARCHETTI et al., 2007).

Recentemente, a transesterificação não catalítica de óleos vegetais usando um álcool em condições supercríticas é estudada, buscando agregar benefícios à qualidade do combustível e ao meio ambiente (DIASAKOU et al., 1998; KUSDIANA; SAKA, 2001a, b, c; DEMIRBAS, 2002; MADRAS et al., 2004; WARABI et al., 2004; DEMIRBAS, 2006). De acordo com a literatura, reações de transesterificação com solventes pressurizados propiciam maior solubilidade entre possíveis fases heterogêneas (FANG et al., 2007; GLISIC et al., 2007; HEGEL et al., 2007), diminuindo as limitações de transporte entre as mesmas, proporcionando maiores taxas de reação, além de simplificar a purificação e separação dos produtos da reação, o que aumenta o interesse nas pesquisas referentes a está metodologia (KUSDIANA; SAKA, 2001a).

A maioria dos relatos na literatura utilizando a transesterificação supercrítica adota o modo batelada para produção de biodiesel. Como principais desvantagens deste processo destacam-se: o longo tempo total de processamento, custo do processo elevado e problemas no controle de qualidade do produto (HE et al., 2007a). Deste modo, a viabilidade de um processo contínuo de transesterificação é de extrema importância a fim de garantir um custo competitivo para o biodiesel (DEMIRBAS, 2006), sendo preferível para aplicação em processos industriais (GOTO et al., 2004), uma vez que propicia um efetivo controle das variáveis de processo.
Com base no contexto descrito, este projeto propôs o estudo de modificações no meio reacional de alcoólise contínua de óleos vegetais em condições pressurizadas, objetivando 0 estabelecimento de condições amenas de operação e garantia de qualidade do produto gerado.

A proposta deste projeto foi viabilizar a obtenção do biodiesel utilizando o óleo extraído da Jatropha curcas L., também conhecida como pinhão manso, através da transesterificação contínua e não-catalítica utilizando etanol em condições pressurizadas. As análises experimentais visaram avaliar a influência das variáveis de processo tais como: temperatura, pressão e tempo de residência sobre a conversão da reação em termos de ésteres etílicos de ácidos graxos, objetivando o estabelecimento de condições amenas de operação e garantia de qualidade do produto gerado. $\mathrm{O}$ método supercrítico apresenta facilidade de separação dos produtos da reação, tornando a purificação do biodiesel mais fácil e sustentável, redução considerável do tempo de reação e não apresenta restrição em relação ao teor de ácidos graxos livres e água presentes nos substratos, justificando a utilização da presente metodologia, para a obtenção de biodiesel proveniente de óleo de pinhão manso.

\section{MATERIAIS E MÉTODOS}

\subsection{Materiais}

Para as reações de transesterificação foram utilizados como substratos óleo de pinhão manso e álcool etílico (Merck 99,9 \% de pureza), sem nenhum tratamento prévio. Para as análises cromatográficas em fase gasosa e etapas de derivatização foram utilizados padrões, reagentes e solventes com procedência Sigma-Aldrich (99,9 $\%$ de pureza). 


\subsection{Descrição do Aparato Experimental}

O sistema utilizado para condução das reações de alcoólise consiste na transesterificação contínua em reator tubular de óleo de pinhão manso, sem uso de catalisador, utilizando álcool etílico em condições pressurizadas.

As análises experimentais para a condução das reações foram realizadas no Laboratório de Tecnologia Supercrítica e Equilíbrio de Fases do Departamento de Engenharia Química da Universidade Estadual de Maringá, sob a orientação do prof. Dr. Lúcio Cardozo Filho e da Me. Simone Belorte de Andrade, tendo como referência o trabalho de Silva (2009). As Figuras 1 e 2 apresentam um diagrama esquemático do aparato experimental, 0 qual consiste de:

C1 - Cilindro de gás. Armazenamento do solvente a ser empregado como co-solvente nos experimentos.

CV - Válvula de Via Única (check-valve, Marca HOKE, Modelo 6133F2Y). A função desta válvula é permitir o fluxo em apenas um sentido.

BS - Bomba de Alta Pressão tipo seringa (Marca ISCO, Modelo 500D) que possui um cilindro interno de $500 \mathrm{~mL}$, pressão de trabalho de até 300 bar e fluxo de até $107 \mathrm{ml} / \mathrm{min}$. O cilindro da bomba é encamisado, o que possibilita a manutenção da temperatura do reservatório em um valor pré-determinado com auxílio de um banho de recirculação;

BR1 - Banho de Recirculação (Marca QUIMIS, Modelo 214M2). Utilizado para manter a temperatura no cilindro da bomba constante;

BI - Bomba de alta pressão de líquidos (HPLC) Digital Série III (Marca Acuflow). Este tipo de bomba opera com vazão constante $(0,1 \mathrm{~mL} / \mathrm{min}$ a $10 \mathrm{~mL} / \mathrm{min}$ ) e pressão de trabalho de 0 a 414 bar. A bomba é utilizada para deslocar a mistura reacional para a zona de reação.
MR - Mistura reacional constituída de óleo de soja e álcool etílico em proporções molares determinadas pelo planejamento de experimentos.

AM - Agitador mecânico (Marca Fisatom, Modelo 712). Inserido em frasco contendo a mistura reacional, utilizado para homogeneizar e manter a mesma sob agitação permanente durante a reação.

RT - Reator tubular, constituído de: tubo sem costura de parede grossa (1/4 in) em aço inox 316 (Marca Sandvik), conexão tipo tubo (Marca Swagelok), conexão tipo $T$ (Marca Swagelok) e zona de pré-aquecimento em tubo (1/16 in) de aço inox 316 (Marca Sandvik). O reator encontrase acoplado a forno a qual fornecia a zona de alta temperatura necessária para reação.

SR - Sistema de resfriamento. Após decorrida a reação, a carga passa por uma banho com água a temperatura ambiente, com o objetivo de cessar a reação e diminuir a temperatura para posterior amostragem.

V1, V2 e V3 - Válvula métrica tipo agulha (Marca HIP, Modelo 15-11AF1, pressão de operação até 1034 bar). A válvula V1 permite o fluxo do solvente do cilindro para a bomba de alta pressão, V2 controla a alimentação ao reator e V3 permite controle da pressão do sistema, amostragem e despressurização.

T1 e T2 - Termopares de isolação mineral tipo $\mathrm{K}$ (Marca Salcas), devidamente calibrados com referência a termopar calibrado por Salcas Laboratório de Termometria, certificado pela RBC (Rede Brasileira de Calibração).

Os termopares se encontram acoplados a entrada e saída do reator, por conexão tipo T (Marca Swagelok), ligados a um sistema de aquisição de dados Field Logger (Marca Novus). 


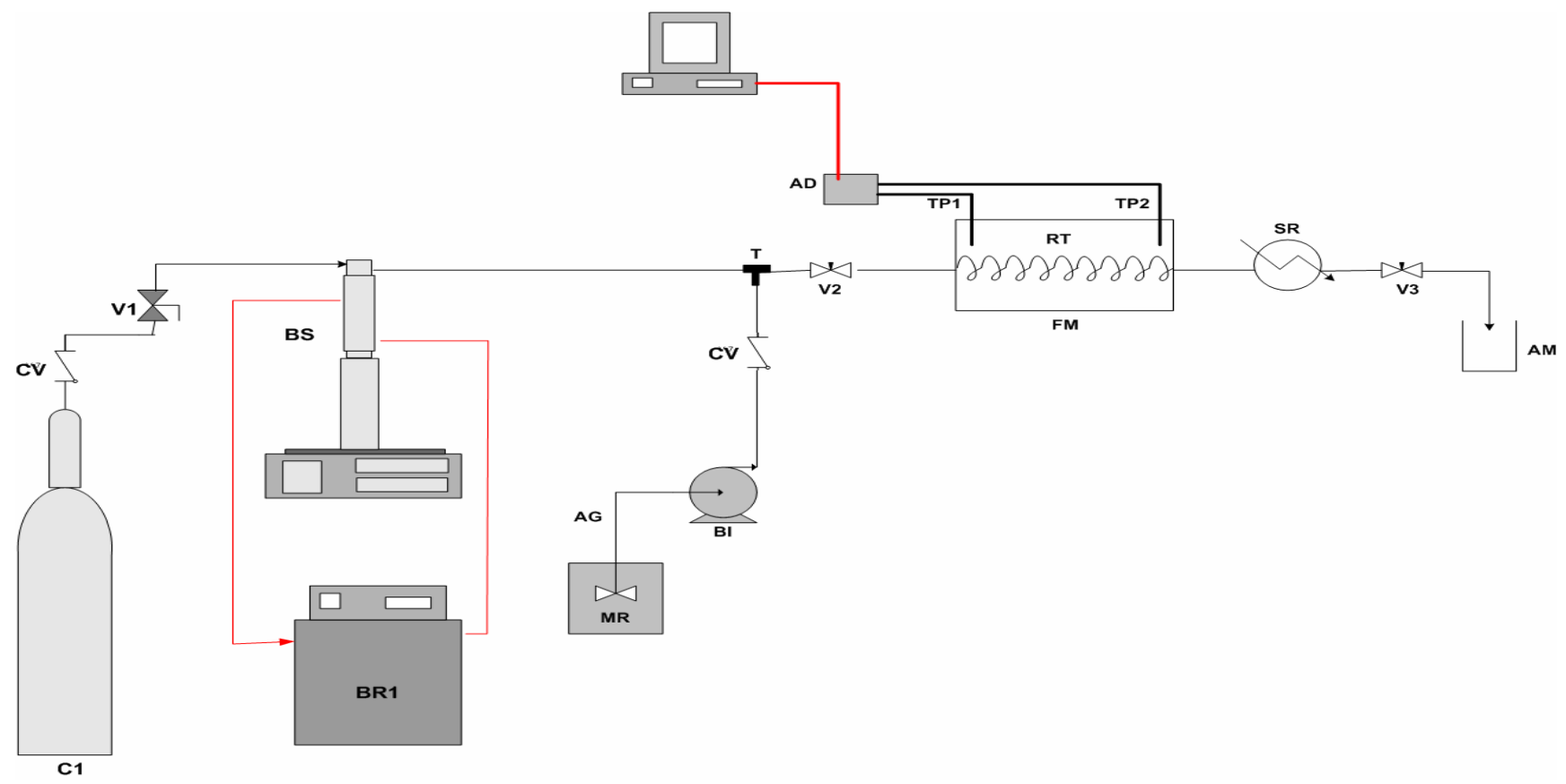

Figura 1. Diagrama esquemático do aparato experimental utilizado nas reações de transesterificação não catalítica: (C1) cilindro de gás, (CV) check-valve, (V1) válvula de controle de fluxo do solvente a bomba de alta pressão, (BS) bomba de alta pressão, (BR1) banho de recirculação, (MR) mistura reacional, (AG) agitador mecânico, (BI) bomba Isocrática, (FM) forno, (RT) reator tubular, (TP1) indicador de temperatura na entrada do reator, (TP2) indicador de temperatura na saída do reator, (AD) Sistema de aquisição de dados, (SR) sistema de resfriamento, (V2) válvula de controle da alimentação, (V3) válvula para controle de pressão e amostragem, (AM) amostragem.

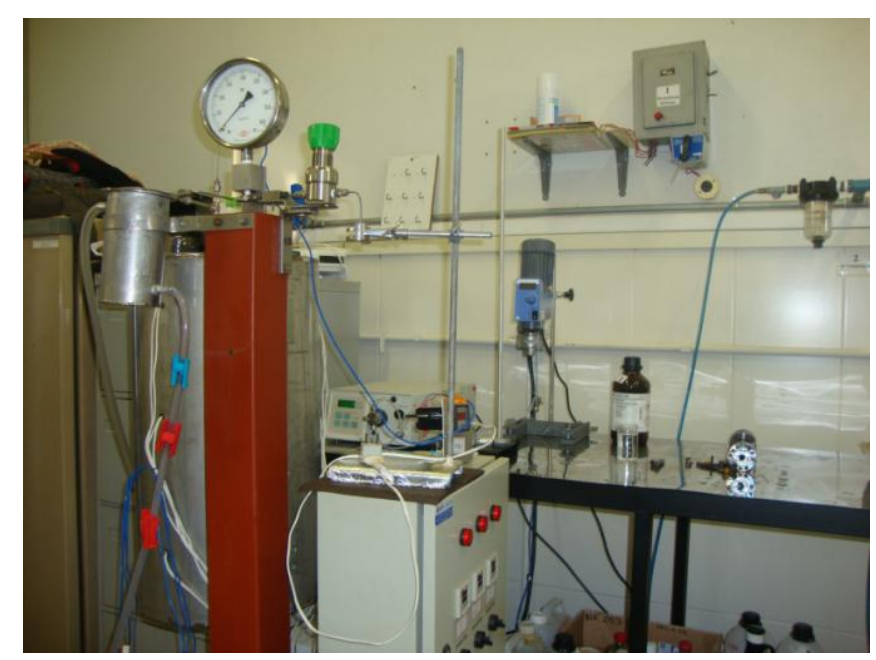

Figura 2. Aparato Experimental Laboratório de Tecnologia Supercrítica e Equilíbrio de Fases do Departamento de Engenharia Química da Universidade Estadual de Maringá. Autor: Lopes e Lima (2012).

\subsection{Procedimento experimental}

O procedimento experimental realizado consistiu no bombeamento contínuo dos substratos óleo vegetal e álcool (previamente homogeneizada por agitador mecânico) a uma dada razão molar (óleo:álcool) e vazão volumétrica de alimentação, até 0 tempo necessário para preencher todo o sistema 
reacional com a mistura. Durante 0 preenchimento do reator, procedeu-se o aquecimento do forno até a temperatura da reação, monitorando-se a temperatura da reação através dos termopares acoplados ao reator, ligados ao indicador de temperatura.

Concomitantemente, 0 banho termostático foi ligado na temperatura de $7^{\circ} \mathrm{C}$, atingida a temperatura iniciou-se o sistema de carga ("refil") na bomba de alta pressão e efetuou-se a abertura do cilindro de solvente $e$ aguardou-se 30 minutos para dispor de maior volume de $\mathrm{CO}_{2}$ na mesma. Depois de decorrido o tempo de carga fechou-se o cilindro e o sistema era então pressurizado da bomba de alta pressão até a válvula de entrada do reator na pressão definida para reação, alterou-se a condição da bomba para vazão constante a uma dada razão de $\mathrm{CO}_{2}$. Todo sistema foi então pressurizado, pelo ajuste da válvula eletropneumática até obtenção da pressão desejada, sendo a mesma monitorada pelo sensor de pressão ligado ao controlador PID.

A carga passou por um sistema de resfriamento e posterior coleta das amostras, que foi realizada em frascos de amostragem (previamente pesados) em triplicata, depois de estabelecidas temperatura e pressão de reação e decorridos dois tempos de residência.

Procedeu-se a evaporação do álcool não reagido das amostras em estufa a vácuo (Marca Quimis, Modelo Q819V2) na temperatura de 65ํำ e 0,05 $\mathrm{MPa}$ de vácuo até obtenção de peso constante.

\subsection{Técnicas Analíticas}

\subsection{1 Ésteres etílicos de ácidos graxos}

Para avaliar a conversão da reação em termos de ésteres, as amostras foram previamente preparadas (após ter evaporado o etanol não reagido), transferindo-se as mesmas do frasco de amostragem para balão volumétrico de $10 \mathrm{~mL}$ com utilização de aproximadamente 2 $\mathrm{mL}$ de álcool etílico e $8 \mathrm{~mL}$ de $\mathrm{n}$-heptano. Após, transferiu-se uma alíquota desta solução para balão de $1 \mathrm{~mL}$, a fim de obter uma concentração de 1000 mg/L e adiciona-se o padrão interno (metil heptadecanoato) na concentração de 250 $\mathrm{mg} / \mathrm{L}$, utilizando como solvente $\mathrm{n}$-heptano.

A solução foi então injetada (1 $\mu L)$ em duplicata em um cromatógrafo gasoso nas condições cromatográficas conforme EN 14103 (2001) possibilitando a determinação do percentual de ésteres etílicos nas amostras e consequentemente 0 valor da conversão da reação, com base a estequiometria da reação.

\subsubsection{Decomposição total da reação}

As amostras condicionadas como descrito anteriormente foram tratadas com $\mathrm{BF}_{3} / \mathrm{MeOH}$ (AOCS, 1990) a fim de derivatizar todos os ácidos graxos (mono-, di- e triglicerídeos, ácidos graxos livres e ésteres etílicos) em seus correspondentes ésteres metílicos, e analisadas conforme condições cromatográficas descritas pela EN 14103 (2001). Para avaliar a porcentagem de decomposição, admitiu-se que o ácido palmítico não é passível de degradação, considerando sua alta estabilidade (HE et al., 2007b; IMAHARA et al., [s.d.]). A decomposição é calculada de acordo com a seguinte equação: 
Decomposição Total $(\%)=100 \times\left[1-\left(\frac{\sum P_{i}}{P_{16: 0}}\right)_{S} \times\left(\frac{P_{16: 0}}{\sum P_{i}}\right)_{O}\right]$

onde, $\sum \mathrm{P}_{\mathrm{i}}$ é o somatório da porcentagem de todos os ésteres etílicos de ácidos graxos, $\mathrm{P}_{16: 0}$ é a porcentagem de C:16, e o "s" e "o" subscritos indicam que as expressões entre ambos serão calculadas considerando a composição do produto das amostras e o óleo original, respectivamente.

\section{RESULTADOS E DISCUSSÃO}

A condução dos experimentos no presente trabalho visaram avaliar a influência das variáveis de processo tais como: temperatura, pressão e tempo de residência sobre a conversão em ésteres etílicos de ácidos graxos.

A razão molar (óleo: etanol) apresenta influência marcante no rendimento em ésteres em reações de transesterificação. A estequiométrica da reação indica uma razão mínima de 1:3 (óleo:etanol), porém reações em condições supercríticas indicam a necessidade da utilização de excesso de etanol a fim de aumentar a solubilidade entre as fases, aumentando a conversão em ésteres. Neste trabalho foi utilizado a razão molar de 1:20, conforme dados obtidos em estudos preliminares e reportados na literatura.

\subsection{Caracterização do óleo de pinhão manso}

A identificação dos constituintes químicos foi realizada empregando-se a cromatografia gasosa associada à espectrometria de massas (GC-MS). A Tabela 01 apresenta as substâncias detectadas no óleo de pinhão manso.
Tabela 1. Composição do óleo de pinhão manso. Autor: Lopes e Lima (2012).

\begin{tabular}{lc}
\hline Componente & Percentual médio \\
\hline C16:0 Ácido Palmítico & 12,7851 \\
C16:1 Ácido & 0,409195 \\
Palmitoléico & 5,581682 \\
C18:0 Ácido Esteárico & 43,58416 \\
C18:1 Ácido Oléico & 37,29466 \\
C18:2 Ácido Linoléico & 0,345194 \\
C18:3 Ácido Linoléico & \\
\hline
\end{tabular}

\subsection{Temperatura de Reação}

Foi utilizada uma faixa de temperatura de $200 \stackrel{\circ}{\circ}$ a $325 \stackrel{\circ}{\circ}$ com o objetivo de avaliar o efeito desta variável sobre o rendimento da reação. Utilizando um tempo de residência de 21 minutos, pode-se observar, conforme Tabela 2, quanto maior a temperatura de reação maior a percentagem de conversão de ácidos graxos em ésteres. Deve-se ressaltar que acima de $325^{\circ} \mathrm{C}$, um decréscimo na conversão de ésteres etílicos do óleo de soja é observado em maiores tempos de reação, comprometendo a estabilidade térmica dos ésteres produzidos, conforme afirmado na bibliografia utilizada.

Tabela 2. Influência da temperatura no rendimento da reação. Autor: Lopes e Lima (2012).

\begin{tabular}{ccccc}
\hline $\mathbf{T}\left({ }^{\circ} \mathbf{C}\right)$ & 250 & 275 & 300 & 325 \\
Rendimento (\%) & 22,27 & 27,90 & 42,15 & 50,02 \\
\hline
\end{tabular}




\subsection{Pressão da Reação}

Para avaliar a influência da pressão do meio reacional sobre o rendimento da reação para produção de biodiesel foram utilizados dois valores de temperatura $\left(300^{\circ} \mathrm{C}\right.$ e $\left.325^{\circ} \mathrm{C}\right)$ e dois valores de pressão (150 bar e 200 bar).

A Tabela 3 apresenta o rendimento da reação em função da pressão.

Tabela 3. Influência da pressão no rendimento da reação. Autor: Lopes e Lima (2012).

\begin{tabular}{ccccc}
\hline & \multicolumn{2}{c}{$\mathbf{T}=\mathbf{3 0 0}^{\circ} \mathbf{C}$} & \multicolumn{2}{c}{$\mathbf{T}=\mathbf{3 2 5}^{\circ} \mathbf{C}$} \\
\hline Pressão (bar) & 150 & 200 & 150 & 200 \\
Rendimento (\%) & 33,03 & 48,76 & 54,90 & 56,38 \\
\hline
\end{tabular}

\subsection{Tempo de residência}

O tempo de residência é fator de extrema importância no rendimento de uma reação. Reações supercríticas se caracterizam pelos altos rendimentos alcançados em baixos tempos de reação. O tempo de residência para reatores tubulares pode ser estimado a partir da razão entre o volume do reator e vazão volumétrica de alimentação dos reagentes, como descrito pela equação abaixo:

$$
\mathrm{t}_{\text {reação }}=\frac{\mathrm{V}}{\mathrm{F}_{\mathrm{MR}}}
$$

onde:

$$
\begin{gathered}
\mathrm{V}=\text { volume de reator }[\mathrm{mL}] ; \\
\mathrm{F}_{\mathrm{MR}}=\text { vazão volumétrica da mistura } \\
\begin{array}{c}
\text { reacional } \\
{[\mathrm{mL} / \mathrm{min}] .}
\end{array}
\end{gathered}
$$

A influência desta variável será estudada pelo ajuste da vazão volumétrica de alimentação da mistura reacional ao reator, a fim de obter diferentes tempos de reação.

Fixando uma temperatura de $300^{\circ} \mathrm{C}$, verificou-se que quanto maior o tempo de reação, maior a percentagem de conversão. A Tabela 4 mostra os resultados variando-se os tempos de reações.

Tabela 4. Influência do tempo de reação no rendimento da reação. Autor: Lopes e Lima (2012).

\begin{tabular}{ccccc}
\hline Tempo (min) & $\mathbf{2 1}$ & $\mathbf{2 8 , 1}$ & $\mathbf{4 2 , 1 5}$ & $\mathbf{5 2 , 7 8}$ \\
Rendimento (\%) & 42,15 & 55,62 & 66,50 & 79,79 \\
\hline
\end{tabular}

\section{CONCLUSÃO}

A partir dos resultados obtidos pode-se concluir que quanto maior a temperatura utilizada, maiores eram as conversões de ácidos graxos em ésteres. É importante ressaltar a impossibilidade de realizar as reações em temperaturas acima de $325^{\circ} \mathrm{C}$, temperatura supercrítica da água, causando a produção de uma borra no reator devido a degradação do etanol, impossibilitando que as reações ocorram.
O tempo de reação também é um fator que influencia na obtenção dos resultados, como verificado, quanto maior o tempo de reação, maiores percentuais de conversão foram obtidos. E quanto maior a pressão no reator, a uma dada temperatura, maiores foram as taxas de conversão da reação em termos de ésteres etílicos de ácidos graxos. 


\section{REFERÊNCIAS}

AKINTAYO, E .T. Characteristics and composition of Parkia biglobbossa and Jatropha curcas oils and cakes. Bioresource Technology, v. 92, p. 307-310, 2004.

AUGUSTUS, G. D. P. S.; JAYABALAN, M.; SEILER, G. J. Evaluation and bioinducion of energy components of Jatropha curcas. Biomass \& Bioenergy, v. 23, p. 161-164, 2002.

ARRUDA, F. P. et al. Cultivo de pinhão-manso (Jatropha curcas L.) como alternativa para 0 semi-árido nordestino. Revista de Oleaginosas e Fibrosas, v. 8, p. 789-799, 2004.

CAO, W.; HAN, H.; ZHANG, J. Preparation of biodiesel from soybean oil using supercritical methanol and $\mathrm{CO}_{2}$ as co-solvent. Process Biochemistry, v. 40, p. 3148-3156, 2005.

CRAWFORD, J. W.; CRAWFORD, J. M.; CRAFTS, R. Transesterification of oil to form biodiesels. United States Patent: 059512, 2007.

DEMIRBAS, A. Biodiesel from vegetable oils via transesterification in supercritical methanol. Energy: Conversion \& Management, v. 43, p. 2349-2356, 2002.

DEMIRBAS, A. Biodiesel production via noncatalytic SCF method and Biodiesel fuel characteristics. Energy Conversion \& Management, v. 47, p. 2271-2282, 2006.

DEMIRBAS, A. Biodiesel from sunflower oil in supercritical methanol with calcium oxide. Energy Conversion \& Management, v. 48, p. 937-941, 2007.

DIASAKOU, M.; LOULOUDI, A.; PAPAYANNAKOS, N. Kinetics of the non-catalytic transesterification of soybean oil. Fuel, v. 77, p. 1297-1302, 1998.

D' IPPOLITO, S. A. et al. Analysis of a two-step, noncatalutic, supercritical biodiesel production process with heat recovery. Energy \& Fuels, v. 21, p. 339-346, 2007.

FANG, T. et al. Supercritical methanol process of modifying oil byproduct for concentrating natural tocopherols. Industrial \& Engineering Chemistry Research, v. 46, p. 5325-5332, 2007.

FOIDL, N. et al. Jatropha curcas L. as a source for the production of biofuel in Nicaragua. Bioresource Technology, v. 58, p. 77-82, 1996.

FUKUDA, H.; KONDO, A.; NODA, H. Biodiesel Fuel Production by Transesterification of Oils.
Journal of Bioscience and Bioengineering, $v$. 92, p. 405-416, 2001.

GLISIC, S. et al. Vapor-liquid equilibria of triglycerides-methanol mixtures and their influence on the Biodiesel synthesis under supercritical conditions of methanol. Journal of the Serbian Chemical Society, v. 72, p. 13-27, 2007.

GOTO, F.; SASAKI, T.; TAKAGI, K. Method and apparatus for preparing fatty acid esters. United States Patent: 6,812,359, 2004.

HE, H.; TAO, W.; ZHU, S. Continuons production of biodiesel from vegetable oil using supercritical methanol process. Fuel, v. 86, p.442-447, 2007a.

HE, H.; TAO, W.; SUN, S. Transesterification kinetics of soybean oil for production of biodiesel in supercritical methanol. JAOCS, v. 84, p. 339404, 2007b.

HEGEL, P. et al. Phase transitions in a biodiesel reactor using supercritical methanol. Industrial \& Engineering Chemistry Research, v.46, p. 6360-6365, 2007.

IMAHARA, $\mathrm{H}$. et al. Thermal stability of biodiesel in supercitical methanol. Fuel (IN PRESS).

KUSDIANA, D.; SAKA, S. Biodiesel fuel from rapeseed oil as prepared in supercritical methanol. Fuel, v. 80, p. 225-231, 2001a.

KUSDIANA, D.; SAKA, S. Kinetics of transesterification in rapeseed oil to Biodiesel fuel as treated in supercritical methanol. Fuel, v. 80, p. 693-698, 2001b.

KUSDIANA, D.; SAKA, S. Methyl Esterification of free fatty acids of rapeseed oil as treated in supercritical methanol. Journal of chemical Engineering of Japan, v. 34, p. 383-387, 2001c.

KUSDIANA, D.; SAKA, S. Two-Step preparation for Catalyst-free biodiesel fuel production. Applied Biochemistry and Biotechonology, v. 113, p. 781-791, 2004a.

MA, F.; HANNA, M. Biodiesel production : a review. Biresource Technology, v. 70, p. 1-15, 1999.

MADRAS, G.; KOLLURU, C.; KUMAR, R. Synthesis of Biodiesel in supercritical fluids. Fuel, v. 83, p. 2029-2033, 2004.

MARCHETTI, J. M.; MIGUEL, V. U.; ERRAZU, A.F. Possible methods for biodiesel production. Renewable \& Sustainable Energy Reviews, v. 11, p. 1300-1311, 2007. 
MINAMI, E.; SAKA, S. Kinetics of hydrolysis and methyl esterification for biodiesel production in two-step supercritical methanol process. Fuel, v. 85, p. 2479-2483, 2006.

OPENSHAW, K. A review of Jatropha curcas: an oil plant of unfulfilled promise. Biomass and Bioenergy, v. 19, p. 1-15, 2000.

PRAMANIK, K. Properties and use of Jatropha curcas oil and diesel fuel blends in compression ignition engine. Renewable Energy, v. 28, p. 239248, 2003.

PINTO, A. C. et al. Biodiesel: An Overview. Journal of the Brazilian Chemical Society, v. 16, p. 1313-1330, 2005.

RAMOS, L.P. et al. Biodiesel: Um projeto de sustentabilidade econômica e sócio-ambinetal para 0 Brasil. Biotecnologia Ciência \& Desenvolvimento, v. 31, p. 27-37, 2003.

REID, R.C.; PRAUSNITZ, J.M.; POLING, B.E. The Properties of gases and liquids. 4. ed. New York: McGraw-Hill Book Company, 1987.

SILVA, C. et al. Continuous production of fatty ethyl esters from soybean oil in compressed ethanol. Industrial \& Engineering Chemistry Research, v. 46, p. 5304-5309, 2007.

SILVA, C. Produção Contínua de Biodiesel por Transesterificação não catalítica de Óleo de Soja. 2009. Tese (Doutorado) - Universidade

SUBRAMANIAN, K. A. et al. Utilization of liquid biofuels in automotive diesel engines: an Indian perspective. Biomass \& Bioenergy, v. 29, p. 6572, 2005.

WARABI, Y., KUSDIANA, D., SAKA, S. Reactivity of triglycerides and fatty acids of rapeseed oil in supercritical alcohols. Bioresource Technology, v. 91, p. 283-287, 2004. 